\title{
On the Strong Coupling Scaling Dimension of High Spin Operators
}

\author{
L. F. Alday ${ }^{a *}$, G. Arutyunov ${ }^{a \dagger}$, M. K. Benna ${ }^{b} \ddagger$ B. Eden ${ }^{a}$, I. R. Klebanov ${ }^{b}$ \\ ${ }^{a}$ Institute for Theoretical Physics and Spinoza Institute, \\ Utrecht University, 3508 TD Utrecht, The Netherlands \\ ${ }^{b}$ Department of Physics and Princeton Center for Theoretical Physics, \\ Princeton University, Princeton, NJ 08544
}

\begin{abstract}
We give an exact analytic solution of the strong coupling limit of the integral equation which was recently proposed to describe the universal scaling function of high spin operators in $\mathcal{N}=4$ gauge theory. The solution agrees with the prediction from string theory, confirms the earlier numerical analysis and provides a basis for developing a systematic perturbation theory around strong coupling.
\end{abstract}

\footnotetext{
*Email: l.f.alday, g.arutyunov, b.eden@phys.uu.nl

${ }^{\dagger}$ Correspondent fellow at Steklov Mathematical Institute, Moscow.

${ }^{\ddagger}$ Email: mbenna, klebanov@princeton.edu
} 


\section{Contents}

1. Introduction 1

2. BES matrix equation at strong coupling 4

2.1 Numerical studies of $\sigma(t)$

2.2 Analytic solution at strong coupling 7

2.3 An alternative derivation of the strong coupling solution 9

2.4 Fluctuation density in the rapidity plane 11

2.5 Subleading corrections 13

3. Leading density from the integral equation 14

4. Conclusions 17

5. Appendix 19

5.1 The inverse Fourier transform of the BES equation 19

5.2 The strong coupling limit 20

\section{Introduction}

The scaling dimension $\Delta$ for twist two operators at large values of the Lorentz spin $S$ is characterized by the universal scaling function (cusp anomalous dimension) $f(g)$ :

$$
\Delta-S=f(g) \ln S+\ldots,
$$

with $g=\sqrt{g_{Y M}^{2} N} / 4 \pi$. The logarithmic dependence of the dimension on large Lorentz spin is a generic feature that has been independently observed for both $\mathcal{N}=4$ SYM and the corresponding string theory dual, see, e.g., [1]-[3]. Importantly, such a scaling behavior stems [4, 5] from the large spin limit of the Bethe equations [6]- [9] which underlie the integrable structures of gauge and string theories. A linear integral equation arising in the large spin limit from the postulated set of gauge/string Bethe equations has been recently derived in [10]. We will refer to it as the BES equation. This equation allows one to compute the universal scaling function $f(g)$ to any desired order in perturbation theory. For instance, for the first few orders one obtains

$$
f(g)=8 g^{2}-\frac{8}{3} \pi^{2} g^{4}+\ldots
$$


Remarkably, independent gauge theory computations [11, 12] confirm this result up to four loop orders, i.e. up to $g^{8}$.

Schematically, the BES equation has the following form

$$
\sigma(t)=1+g^{2} \int K\left(t, t^{\prime}\right) \sigma\left(t^{\prime}\right) d t^{\prime}
$$

where $K\left(t, t^{\prime}\right)=K^{(m)}\left(t, t^{\prime}\right)+\kappa K^{(c)}\left(t, t^{\prime}\right)$ and $\kappa=2$. Here $K^{(m)}$ is the so-called main scattering kernel and $K^{(c)}$ is the dressing kernel. The dressing kernel arises upon taking into account an additional scattering phase in the S-matrix which is apparently needed to achieve an agreement with string theory predictions in the strong coupling limit [7]. This phase is also the only freedom in the S-matrix which cannot be fixed by symmetry arguments [13]. ${ }^{1}$ The form of the phase is largely but not completely constrained by an additional requirement of crossing symmetry [17]. The constraints arising from crossing symmetry were shown to hold [18] at two leading orders in string perturbation theory [0, 19]. Recently an interesting solution for the crossing symmetric phase has been obtained [20] which agrees nicely with all available string and field-theoretic data.

The agreement observed at the four loop level between the scaling function of BES and the results obtained in the field-theoretic framework is very important, because it implies the appearance of the additional scattering phase in perturbation theory and, therefore, it distinguishes the BES equation from the earlier proposal [5] which corresponds to taking $\kappa=0$.

According to the AdS/CFT duality [21] the large $g$ behavior of $f(g)$ can be extracted by studying the energy of the folded string spinning in the $\mathrm{AdS}_{3}$ part of the target space (the GKP solution) and it turns out to be [2, 3]

$$
f(g)=4 g-\frac{3 \ln 2}{\pi}+\ldots
$$

In the recent work [22] and 223] an important question was addressed, namely, how the perturbative expansion controlled by the BES equation can be extrapolated to large values of the coupling constant and wether the resulting expression is consistent with the string theory predictions. In particular, in [22] a numerical method was devised to solve the BES equation around the strong coupling point and numerical solutions were shown to be in perfect agreement with eq. (1.1), providing a non-trivial test of the AdS/CFT correspondence.

In this note we find an exact analytic expression for the density $\sigma(t)$ in the strong coupling limit. If one insists on truncating the BES equation at leading order for large $g$ the kernel becomes degenerate and therefore a unique solution for $\sigma(t)$ cannot be found without additional assumptions. However, for finite values of $g$ the solution

\footnotetext{
${ }^{1}$ See [14]-[16] for recent studies of the string S-matrix and its symmetries.
} 
is unique and can be found numerically with high precision. These two statements are consistent because expanding the BES equation in a power series in $1 / g$ we find a second equation at subleading order that removes any ambiguity in the strong coupling solution $\sigma(t)$.

To gain some intuition our approach will be to first investigate the solution numerically for finite values of the coupling, which will suggest a simple additional requirement that should be imposed on the underdetermined, truncated BES equation to single out a unique solution in the strong coupling limit which is the convergence point of our numerics. After this additional requirement is identified, we find an analytic solution for $\sigma(t)$ in the strong coupling limit. We then show that this solution is in fact completely determined by taking into account the subleading contributions to the BES equation, thus confirming analytically the validity of the auxiliary condition suggested by the numerics and of our strong coupling solution $\sigma(t)$.

Upon making the Fourier transform to the rapidity $u$-plane the corresponding density $\sigma(u)$ reads

$$
\sigma(u)=\frac{1}{4 \pi g^{2}}\left(1-\frac{\theta(|u|-1)}{\sqrt{2}} \sqrt{1+\frac{1}{\sqrt{1-\frac{1}{u^{2}}}}}\right),
$$

where $\theta(u)$ is the step function. Thus, on the rapidity plane the leading density is an algebraic function which is constant in the interval $|u|<1$. We see that, in contrast to the weak coupling solution of the BES equation [10], the strong coupling density exhibits a gap between $[-\infty,-1]$ and $[1, \infty]$, see Fig.1. Remarkably, this is reminiscent of the behavior of the corresponding solutions describing classical spinning strings.

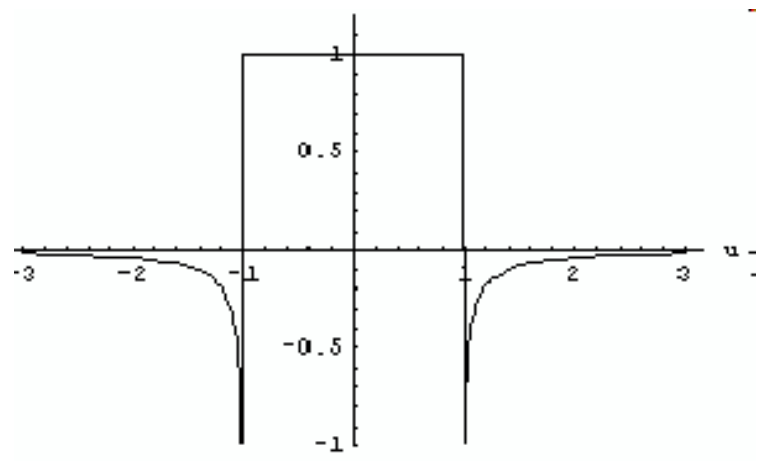

Fig.1. The plot of the analytic solution for the leading density $4 \pi g^{2} \sigma(u)$.

We also would like to point out that the strong coupling solution of the BES equation we have found is apparently different from the singular solution $\chi_{\text {sing }}$ discussed in [23]. 
The paper is organized as follows. In section 2 we analyze the matrix form of the BES equation at strong coupling, first numerically and then analytically. We compute the coefficients in the expansion of $\sigma(t)$ in terms of Bessel functions and based on this numerical analysis we make a guess of how these coefficients could be (partially) related to each other at strong coupling. In the strong coupling limit we obtain an analytic equation for the coefficients which turns out to have a degenerate kernel. Supplementing this equation with the proposed relation among the coefficients allows us to find an exact analytical solution for $\sigma(t)$. We then show that in fact no guess is necessary if one expands the BES equation to higher order in $1 / g$, which leads to a second equation that singles out our solution as the correct one.

We proceed to find an analogous pair of equations for the subleading coefficients at strong coupling. Again they appear at different orders in the inverse coupling constant, each of them individually being a degenerate, half-rank equation. We identify some constraints on the subleading value of $\sigma(t)$, but postpone a detailed analysis of the strong coupling perturbation theory to a future publication. In section 3 we analyze the BES equation at strong coupling in the Fourier space and verify that the results of section 2 agree with this analysis. Finally, we end with conclusions and open problems.

\section{BES matrix equation at strong coupling}

In this section we study the density of fluctuations $\sigma(t)$ for large values of the coupling constant. First we argue, by performing numerical analysis, that $\sigma(t)$ obeys a certain additional requirement. Then we consider the matrix BES equation at leading order in the large $g$ expansion, use the previously found requirement to solve for the density and confirm by expanding the BES equation further that this solution is in fact complete determined analytically. Finally, we derive and briefly discuss constraints on the subleading value of $\sigma(t)$.

\subsection{Numerical studies of $\sigma(t)$}

The universal scaling function is related to $\sigma(t)$ as follows

$$
f(g)=16 g^{2} \sigma(0)
$$

where the density of fluctuations $\sigma(t)$ satisfies the following integral equation [5, 10]

$$
\sigma(t)=\frac{t}{e^{t}-1}\left(K(2 g t, 0)-4 g^{2} \int_{0}^{\infty} \mathrm{d} t^{\prime} K\left(2 g t, 2 g t^{\prime}\right) \sigma\left(t^{\prime}\right)\right) .
$$

The kernel separates into two pieces, the main scattering kernel $K^{(m)}$ and the dressing kernel $K^{(c)}$

$$
K\left(t, t^{\prime}\right)=K^{(m)}\left(t, t^{\prime}\right)+2 K^{(c)}\left(t, t^{\prime}\right),
$$


which are expressed in terms of Bessel functions as follows

$$
\begin{aligned}
K^{(m)}\left(t, t^{\prime}\right) & =\frac{J_{1}(t) J_{0}\left(t^{\prime}\right)-J_{0}(t) J_{1}\left(t^{\prime}\right)}{t-t^{\prime}} \\
K^{(c)}\left(t, t^{\prime}\right) & =4 g^{2} \int_{0}^{\infty} \mathrm{d} t^{\prime \prime} K_{1}\left(t, 2 g t^{\prime \prime}\right) \frac{t^{\prime \prime}}{e^{t^{\prime \prime}}-1} K_{0}\left(2 g t^{\prime \prime}, t^{\prime}\right) .
\end{aligned}
$$

Here $K_{0}$ and $K_{1}$ denote even and odd parts of the kernel under change of the sign of the arguments:

$$
\begin{aligned}
& K_{0}\left(t, t^{\prime}\right)=\frac{t J_{1}(t) J_{0}\left(t^{\prime}\right)-t^{\prime} J_{0}(t) J_{1}\left(t^{\prime}\right)}{t^{2}-t^{\prime 2}}=\frac{2}{t t^{\prime}} \sum_{n=1}^{\infty}(2 n-1) J_{2 n-1}(t) J_{2 n-1}\left(t^{\prime}\right), \\
& K_{1}\left(t, t^{\prime}\right)=\frac{t^{\prime} J_{1}(t) J_{0}\left(t^{\prime}\right)-t J_{0}(t) J_{1}\left(t^{\prime}\right)}{t^{2}-t^{\prime 2}}=\frac{2}{t t^{\prime}} \sum_{n=1}^{\infty} 2 n J_{2 n}(t) J_{2 n}\left(t^{\prime}\right) .
\end{aligned}
$$

As was shown in [22], if one introduces a function

$$
s(t)=\frac{e^{t}-1}{t} \sigma(t)
$$

and expands $s(t)$ in the following series

$$
s(t)=\sum_{n=1}^{\infty} s_{n} \frac{J_{n}(2 g t)}{2 g t}
$$

then eq.(2.2) transforms into a matrix equation for the vector $s=\left(s_{1}, s_{2}, s_{3}, \ldots\right)^{t}$

$$
\left(1+K^{(m)}+2 K^{(c)}\right) s=(1+2 C) e .
$$

Here $e=(1,0,0,0, \ldots)^{t}$ and $K^{(m)}, K^{(c)}, C$ are infinite-dimensional matrices with the entries

$$
\begin{aligned}
K_{m n}^{(m)} & =2(N Z)_{m n} \\
K_{m n}^{(c)} & =2(C Z)_{m n} \\
C_{m n} & =2(P N Z Q N)_{m n}
\end{aligned}
$$

where $Q=\operatorname{diag}(1,0,1,0, \ldots), P=\operatorname{diag}(0,1,0,1, \ldots)$ and $N=\operatorname{diag}(1,2,3, \ldots)$. The coupling constant enters into the matrix $Z$ whose entries are given by

$$
Z_{m n}=\int_{0}^{\infty} \mathrm{d} t \frac{J_{m}(2 g t) J_{n}(2 g t)}{t\left(e^{t}-1\right)} .
$$

The key observation is that for intermediate values of $g$, for instance $2<g<20$, one can approximate the infinite-dimensional matrices entering the BES equation by matrices of finite rank $d$, with $d$ not much larger than $g$. With matrices of finite 
rank it is possible to solve numerically for the coefficients $s_{k}$ for different values of the coupling constant and to find the best fit result for an expansion of the type

$$
s_{k}=\frac{1}{g} s_{k}^{\ell}+\frac{1}{g^{2}} s_{k}^{s \ell}+\ldots
$$

As the numerical analysis indicates, the finite rank approximation is valid for computing the coefficients $s_{k}$ with $k \ll d$. We have solved numerically eq.(2.7) for numerous points in the range $2<g<20$ and use $d=50$. In the table below the values for a few leading coefficients $s_{k}^{\ell}$ are exhibited.

\begin{tabular}{|c|c|c|c|}
\hline$k$ & $s_{2 k-1}^{\ell}$ & $s_{2 k}^{\ell}$ & $100\left|\left(s_{2 k-1}^{\ell}-s_{2 k}^{\ell}\right) / s_{2 k}^{\ell}\right|$ \\
\hline 1 & 0.500006 & 0.499993 & 0.003 \\
2 & -0.75005 & -0.74977 & 0.038 \\
3 & 0.93727 & 0.93676 & 0.055 \\
4 & -1.09281 & -1.09415 & 0.12 \\
5 & 1.2239 & 1.2333 & 0.77 \\
\hline
\end{tabular}

Some comments are in order. First, notice that the value for $s_{1}^{\ell}$ is in perfect agreement with the value predicted from string theory $s_{1}^{\ell}=1 / 2$ and confirmed numerically (with a precision higher than the one presented here) by [22]. Second, notice that the difference between $s_{2 k-1}^{\ell}$ and $s_{2 k}^{\ell}$ is in all the cases smaller than $1 \%$, and gets bigger as $k$ increases; this is related to the fact that the rank of the matrices is finite.

Thus, the numerical analysis suggests that in the limit of infinite rank matrices the following relation holds for the leading coefficients in the strong coupling expansion

$$
s_{2 k-1}^{\ell}=s_{2 k}^{\ell}
$$

As we will see later on, this condition will allow one to solve analytically for the coefficients $s_{k}^{\ell}$ and the values obtained will be in perfect agreement with the ones computed numerically.

Further evidence comes from the fact that one can approximate the matrix elements $Z_{m n}$ by their analytic values at strong coupling (see next subsection). Therefore, one can consider matrices of much higher rank, fix a sufficiently large value of $g$ and compute (numerically) the coefficients $s_{k}^{\ell}$. Below we present the results for $g=10000$ and $d=250$.

\begin{tabular}{|c|c|c|c|}
\hline$k$ & $s_{2 k-1}^{\ell}$ & $s_{2 k}^{\ell}$ & $100\left|\left(s_{2 k-1}^{\ell}-s_{2 k}^{\ell}\right) / s_{2 k}^{\ell}\right|$ \\
\hline 1 & 0.49993 & 0.49991 & 0.0049 \\
2 & -0.74943 & -0.74938 & 0.0073 \\
3 & 0.93585 & 0.93577 & 0.0085 \\
4 & -1.09033 & -1.09023 & 0.0089 \\
5 & 1.22455 & 1.22444 & 0.0087 \\
\hline
\end{tabular}


As $d$ increases, we see that the difference between $s_{2 k-1}^{\ell}$ and $s_{2 k}^{\ell}$ (for $k=2,3,4$ ) decreases considerably. Also, the difference is approximately constant for small values of $k$. We should stress, however, that a priory there is no reason to expect the results obtained by keeping in the BES equation only the leading term for $Z$ to be valid, since as we will see the subleading terms in the matrix elements $Z_{m n}$ are necessary to fix uniquely the leading order solution for $s_{k}$. Surprisingly, one still obtains a good approximation to the large $g$ solution in this fashion. Nevertheless, we regard the present computation as less robust.

To conclude, requiring continuity in $g$, the leading coefficients $s_{k}^{\ell}$ in the strong coupling expansion of the function $\sigma(t)$ exhibit the relation (2.10), which constraints a possible form of $\sigma(t)$.

\subsection{Analytic solution at strong coupling}

For finite real values of $g$ the matrix element $Z_{m n}$ is given by a convergent integral. However, it is not obvious if it is possible to express the result of integration as a power series in $1 / g$. Indeed, expanding the integrand in eq.(2.8) as

$$
Z_{m n}=\int_{0}^{\infty} \mathrm{d} t \frac{J_{m}(t) J_{n}(t)}{t}\left(\frac{2 g}{t}-\frac{1}{2}+\frac{t}{24 g}+\ldots\right),
$$

leads to a power series (with coefficients given essentially by the Bernoulli numbers) which converges only for $|t /(2 g)|<2 \pi$. We see that only the first two leading terms in this expansion can be integrated, while already in the third term divergent integrals appear. It is therefore natural to assume that the expansion of $Z$ develops as

$$
Z=g Z^{\ell}+Z^{s \ell}+\ldots
$$

where the first two terms, $Z^{\ell}$ and $Z^{\text {sl}}$, are given by the convergent integrals mentioned above. Explicitly, they are

$$
\begin{aligned}
Z_{m n}^{\ell} & =-\frac{8}{\pi} \frac{\cos ((m-n) \pi / 2)}{(m+n+1)(m+n-1)(m-n+1)(m-n-1)}, \\
Z_{m n}^{s \ell} & =-\frac{1}{\pi} \frac{\sin ((m-n) \pi / 2)}{m^{2}-n^{2}} .
\end{aligned}
$$

Assumption (2.11) is well supported by the numerics. We have checked that numerical values for $Z_{m n}$ for large $g$ are in a very good agreement with the analytic expressions (2.12). On the other hand, the status of the higher order terms in eq.(2.11) is not clear to $\mathrm{us}^{2}$. Thus, in what follows, we largely restrict our investigation of the BES equation to the first two leading terms.

\footnotetext{
${ }^{2}$ For instance, whether expansion (2.11) contains terms of the type $\log (g) / g$, etc. From the string theory point of view, the appearance of such terms could be an indication of a two-loop divergence of the world-sheet S-matrix. Further analysis is needed to clarify this issue.
} 
With this word of caution we proceed to investigate eq. (2.7) in the strong coupling limit. It is not hard to see that it implies the following equation for the leading vector $s^{\ell}$

$$
K^{(c) \ell} s^{\ell}=C^{\ell} e
$$

where the leading matrices $K^{(c) \ell}$ and $C^{\ell}$ are obtained by keeping the leading contribution $Z^{\ell}$ only. It turns out that for even values of $d$ the kernel $K^{(c) \ell}$ has rank $d / 2$, hence from the equation above it is possible to solve only for half of the components of $s$. However, now the condition (2.10) enters into play. Imposing eq.(2.10) together with eq.(2.13) allows one to uniquely determine the vector $s^{\ell}$.

In order to find the solution in the limit of infinite $d$ it is convenient to express the leading equation in the following way

$$
K^{o} s^{o}+K^{e} s^{e}=\frac{1}{2} e,
$$

where $s^{o}, s^{e}$ are vectors of length $d / 2$ comprising the odd and even components of $s^{\ell}$ respectively: $s^{o}=\left(s_{1}^{\ell}, s_{3}^{\ell}, s_{5}^{\ell}, \ldots\right)^{t}$ and $s^{e}=\left(s_{2}^{\ell}, s_{4}^{\ell}, s_{6}^{\ell}, \ldots\right)^{t}$. Further $e=(1,0,0, \ldots)^{t}$. It is easy to check that eqs. 2.14) and (2.13) are equivalent provided

$$
\left(K^{o}\right)_{m n}=Z_{2 m-1,2 n-1}^{\ell}+Z_{2 m+1,2 n-1}^{\ell}, \quad\left(K^{e}\right)_{m n}=Z_{2 m-1,2 n}^{\ell}+Z_{2 m+1,2 n}^{\ell} .
$$

Then, we find that the unique solution of eq.(2.14) satisfying the relation $s^{o}=s^{e}$ turns out to be

$$
s_{2 k-1}^{\ell}=s_{2 k}^{\ell}=(-1)^{k+1} \frac{\Gamma\left(k+\frac{1}{2}\right)}{\Gamma(k) \Gamma\left(\frac{1}{2}\right)} .
$$

This remarkably simple expression for the coefficients $s_{k}^{\ell}$ is the main result of this paper. By using the following identities (true in the limit of infinite rank)

$$
\begin{aligned}
\left(K^{e-1}\right)_{m n} & =-4(-1)^{m+n+1} m n^{2}, \quad n \leq m \\
& =-4(-1)^{m+n+1} m^{3}, \quad n>m \\
\left(K^{e-1} K^{o}\right)_{m n} & =(-1)^{m-n} \frac{32}{\pi} \frac{m^{3}}{\left(4 m^{2}-(1-2 n)^{2}\right)(1-2 n)^{2}}
\end{aligned}
$$

one can check explicitly that the coefficients (2.16) indeed solve eq.(2.14). Thus, we found that restricting the coefficients $s_{k}$ to the leading order expressions $s_{k}^{\ell}$ the function $s(t)$ is given by

$$
s(t)=\frac{1}{g} \sum_{k=1}^{\infty}(-1)^{k+1} \frac{\Gamma\left(k+\frac{1}{2}\right)}{\Gamma(k) \Gamma\left(\frac{1}{2}\right)} \frac{J_{2 k}(2 g t)+J_{2 k-1}(2 g t)}{2 g t},
$$

where we have omitted the subleading contributions. The last formula can be considered as the leading term in the large $g$ expansion of the density $s(t, g)$ with $g t$ kept finite. As is clear from eq.(2.2), we are only interested in values of $s(t)$ for $t \geq 0$. 
The series can be summed and for this range of $t$ the result expressed in terms of the confluent hypergeometric function of the second kind $U(a, b, x)$ :

$$
\begin{aligned}
s(t)= & -\frac{i}{8 \pi g^{2} t} e^{2 i g t}\left(\Gamma\left(\frac{3}{4}\right) U\left(-\frac{1}{4}, 0,-4 i g t\right)+\Gamma\left(\frac{5}{4}\right) U\left(\frac{1}{4}, 0,-4 i g t\right)\right) \\
& +\frac{i}{8 \pi g^{2} t} e^{-2 i g t}\left(\Gamma\left(\frac{3}{4}\right) U\left(-\frac{1}{4}, 0,4 i g t\right)+\Gamma\left(\frac{5}{4}\right) U\left(\frac{1}{4}, 0,4 i g t\right)\right) .
\end{aligned}
$$

The leading density (2.19) has a rather complicated profile. For $g t \rightarrow 0$ it perfectly reproduces the desired result $s(t) \rightarrow 1 / 4 g$. On the other hand, the asymptotic of $s(t)$ for $g t \rightarrow \infty$ exhibits a highly oscillating behavior.

\subsection{An alternative derivation of the strong coupling solution}

Let us now show that the result (2.16) can in fact be derived without resorting to any auxiliary conditions obtained from numerical arguments. The degenerate equation appearing at leading order, which can be used to express one half of the coefficients $s_{n}$ in terms of the other, can be supplemented by another half-rank equation from subleading terms in the BES equation. Together they determine a unique solution.

Writing out the integral equation (2.2) in the basis (2.6) with explicit matrix indices we find

$$
\begin{aligned}
s_{n} \frac{J_{n}(2 g t)}{2 g t}= & \frac{J_{1}(2 g t)}{2 g t}+8 n Z_{2 n, 1} \frac{J_{2 n}(2 g t)}{2 g t}-2 n Z_{n m} s_{m} \frac{J_{n}(2 g t)}{2 g t} \\
& -16 n(2 m-1) Z_{2 n, 2 m-1} Z_{2 m-1, r} s_{r} \frac{J_{2 n}(2 g t)}{2 g t}
\end{aligned}
$$

where all indices are summed over from 1 to $\infty$. Now we split up the integral equation according to powers of $g$ and into odd and even rows (indices of Bessel functions).

At $\mathcal{O}(g)$ the odd equation is trivial and the even one reads:

$$
2(2 m-1) Z_{2 n, 2 m-1}^{\ell} Z_{2 m-1, r}^{\ell} s_{r}^{\ell}=Z_{2 n, 1}^{\ell}=\frac{1}{4} \delta_{n, 1} .
$$

This is precisely eq.(2.13) employed in the previous subsection. At $\mathcal{O}(1)$ the odd rows lead to the condition

$$
Z_{2 m-1, r}^{\ell} s_{r}^{\ell}=\frac{1}{2} \delta_{m, 1}
$$

Actually this equation implies the previous one. It determines one half of the coefficients $s_{n}^{\ell}$ in terms of the other. Expanding further, at $\mathcal{O}(1)$ the even equation is given by

$$
\begin{aligned}
& 8 n Z_{2 n, 1}^{s \ell}-4 n Z_{2 n, m}^{\ell} s_{m}^{\ell}-16 n(2 m-1) Z_{2 n, 2 m-1}^{\ell} Z_{2 m-1, r}^{\ell} s_{r}^{s \ell} \\
- & 16 n(2 m-1) Z_{2 n, 2 m-1}^{\ell} Z_{2 m-1, r}^{s \ell} s_{r}^{\ell}-16 n(2 m-1) Z_{2 n, 2 m-1}^{s \ell} Z_{2 m-1, r}^{\ell} s_{r}^{\ell}=0 .
\end{aligned}
$$


To determine the other half of the coefficients $s_{n}^{\ell}$ we need to eliminate $s_{n}^{s \ell}$ from this equation. To do this we examine the odd equation at $\mathcal{O}(1 / g)$

$$
-2(2 m-1) Z_{2 m-1, r}^{\ell} s_{r}^{s \ell}-2(2 m-1) Z_{2 m-1, r}^{s \ell} s_{r}^{\ell}=s_{2 m-1}^{\ell} .
$$

Now we use (2.22) and (2.24) to simplify (2.23), which gives

$$
Z_{2 n, m}^{\ell} s_{m}^{\ell}-2 Z_{2 n, 2 m-1}^{\ell} s_{2 m-1}^{\ell}=Z_{2 n, m}^{\ell}(-1)^{m} s_{m}^{\ell}=0 .
$$

This is the second equation we were looking for, which together with (2.22) completely determines $s_{n}^{\ell}$. If we define a matrix $\tilde{Z}_{n m}$ which is identical to $Z_{n m}$ except for a sign flip when both $n$ is even and $m$ is odd, we can combine the two conditions into the full-rank equation for the strong coupling solution

$$
\tilde{Z}_{n m}^{\ell} s_{m}^{\ell}=\frac{1}{2} \delta_{n, 1} .
$$

Note that the additional minus signs in the definition of $\tilde{Z}_{n m}$ arise precisely because of the introduction of the dressing kernel, and would by absent for $\kappa=0$. Writing out (2.26) explicitly shows that the $s_{n}^{\ell}$ have to satisfy

$$
\begin{aligned}
& \sum_{k=1}^{\infty} \frac{4(-1)^{n+k+1} s_{2 k-1}^{\ell}}{(2 n-2 k-1)(2 n-2 k+1)(2 n+2 k-3)(2 n+2 k-1) \pi} \\
& =-\frac{s_{2 n}^{\ell}}{8 n(2 n-1)}-\frac{s_{2 n-2}^{\ell}}{8(n-1)(2 n-1)}+\frac{1}{4} \delta_{n, 1}, \\
& \sum_{k=1}^{\infty} \frac{4(-1)^{n+k+1} s_{2 k}^{\ell}}{(2 n-2 k-1)(2 n-2 k+1)(2 n+2 k-1)(2 n+2 k+1) \pi} \\
& =\frac{s_{2 n+1}^{\ell}}{8 n(2 n+1)}+\frac{s_{2 n-1}^{\ell}}{8 n(2 n-1)},
\end{aligned}
$$

for all $n \geq 1$ (where it is understood that the second term on the right hand side of (2.27) is absent for $n=1$ ). Indeed these equations are obeyed by

$$
s_{2 n-1}^{\ell}=s_{2 n}^{\ell}=\frac{(-1)^{n-1}(2 n-1) ! !}{2^{n}(n-1) !},
$$

which is precisely the solution (2.16) found in the previous subsection. To show this, note that the coefficients $s_{2 n-1}^{\ell}$ are generated by the Taylor expansion of $(1+x)^{-3 / 2}$, which makes it easy to perform the above sums as integrals over functions of the form $x^{m}\left(1-x^{2}\right)^{-3 / 2}$ for some appropriate power $m$ chosen to generate the necessary terms in the denominators of the left hand sides of eqs.(2.27) and (2.28). 


\subsection{Fluctuation density in the rapidity plane}

To get more insight into the structure of the leading solution, we find it convenient to perform the (inverse) Fourier transform of the density $\sigma(t) \rightarrow \sigma(u)$ :

$$
\sigma(u)=\frac{1}{2 \pi} \int_{-\infty}^{\infty} \mathrm{d} t e^{i 2 g t u} e^{-|t| / 2} \sigma(|t|) .
$$

We recall that $u$ is a rapidity variable originally used to parameterize the Bethe root distributions of gauge and string theory Bethe ansätze [6, 7], which gives another reason for studying the density of fluctuations on the $u$-plane. Thus, substituting in eq.(2.30) the power series expansion for $\sigma(t)$ we get

$$
\sigma(u)=\frac{1}{2 \pi} \sum_{n=1}^{\infty} s_{n} \int_{-\infty}^{\infty} \mathrm{d} t e^{i 2 g t u} e^{-|t| / 2} \frac{|t|}{e^{|t|}-1} \frac{J_{n}(2 g|t|)}{2 g|t|}+\ldots
$$

For large values of $g$ this expression can be well approximated as

$$
\sigma(u)=\frac{1}{2 \pi} \sum_{n=1}^{\infty} s_{n} \int_{-\infty}^{\infty} \mathrm{d} t e^{i 2 g t u} e^{-|t| / 2} \frac{J_{n}(2 g|t|)}{2 g|t|}+\ldots
$$

The last integral is computed by using the following formula [5]

$$
\int_{0}^{\infty} \mathrm{d} t e^{ \pm 2 g i u t} e^{-t / 2} \frac{J_{n}(2 g t)}{2 g t}=\frac{(2 g)^{n-1}}{n}\left(u^{ \pm}\left(1+\sqrt{1+4 g^{2} /\left(u^{ \pm}\right)^{2}}\right)\right)^{-n}
$$

with $u^{ \pm}=1 / 2 \mp 2 i g u$.

In this way we obtain the following series representation for the density $\sigma(u)$

$$
\sigma(u)=\frac{1}{2 \pi g} \sum_{n=1}^{\infty} s_{n} f_{n}+\ldots,
$$

where

$$
f_{n}=\frac{(2 g)^{n}}{2 n}\left[\left(u^{+}\left(1+\sqrt{1+4 g^{2} /\left(u^{+}\right)^{2}}\right)\right)^{-n}+\left(u^{-}\left(1+\sqrt{1+4 g^{2} /\left(u^{-}\right)^{2}}\right)\right)^{-n}\right] .
$$

In what follows it is convenient to introduce the expansion parameter $\epsilon=1 /(2 g)$. Our considerations above suggest that the density $\sigma(u)$ expands starting from the second order in $\epsilon$ :

$$
\sigma(u)=\epsilon^{2} \sigma^{\ell}(u)+\epsilon^{3} \sigma^{s \ell}(u)+\ldots
$$

To find the leading contribution $\sigma^{\ell}(u)$ we have to develop the large $g$ expansion of the functions $f_{n}$. The result is not uniform, it depends on whether $n$ is even or odd and also on the value of $u$. For $n$ even we find

$$
f_{2 k}=\left\{\begin{array}{l}
\frac{(-1)^{k}}{2 k} T_{2 k}(u)+\mathcal{O}(\epsilon) \quad \text { for } \quad|u|<1, \\
\frac{(-1)^{k}}{2 k}\left(u\left(1+\sqrt{1-\frac{1}{u^{2}}}\right)\right)^{-2 k}+\mathcal{O}(\epsilon) \quad \text { for } \quad|u|>1 .
\end{array}\right.
$$


Here $T_{2 k}(u)$ are the Chebyshev polynomials of the first kind. For $n$ odd we obtain

$$
f_{2 k-1}=\left\{\begin{array}{c}
-\frac{(-1)^{k}}{2 k-1} \sqrt{1-u^{2}} U_{2 k-2}(u)+\mathcal{O}(\epsilon) \quad \text { for } \quad|u|<1, \\
0+\mathcal{O}(\epsilon) \quad \text { for } \quad|u|>1
\end{array}\right.
$$

Here $U_{2 k-2}(u)$ are the Chebyshev polynomials of the second kind. We recall that the Chebyshev polynomials of the first and the second kind form a sequence of orthogonal polynomials on the interval $[-1,1]$ with the weights $\left(1-u^{2}\right)^{-1 / 2}$ and $\left(1-u^{2}\right)^{1 / 2}$ respectively.

To find the leading density $\sigma^{\ell}(u)$ inside the interval $|u|<1$ we can use the trigonometric definition of the Chebyshev polynomials which corresponds to choosing parametrization $u=\cos \theta$ with $0 \leq \theta \leq \pi$. Thus, taking the limit $g \rightarrow \infty$ we obtain for the leading density the following expression

$$
\sigma^{\ell}(u)=\frac{2}{\pi} \sum_{k=1}^{\infty}\left(s_{2 k-1}^{\ell} f_{2 k-1}(\theta)+s_{2 k}^{\ell} f_{2 k}(\theta)\right),
$$

where

$$
f_{2 k}(\theta)=(-1)^{k} \frac{\cos 2 k \theta}{2 k}, \quad f_{2 k-1}(\theta)=-(-1)^{k} \frac{\sin (2 k-1) \theta}{2 k-1} .
$$

Given the proposal (2.16) for the coefficients $s_{k}^{\ell}$, we can now sum the series for $\sigma^{\ell}(u)$ and obtain

$$
\sigma^{\ell}(u)=\frac{1}{\pi}
$$

Thus, inside the interval $[-1,1]$ the density $\sigma^{\ell}(u)$ is constant.
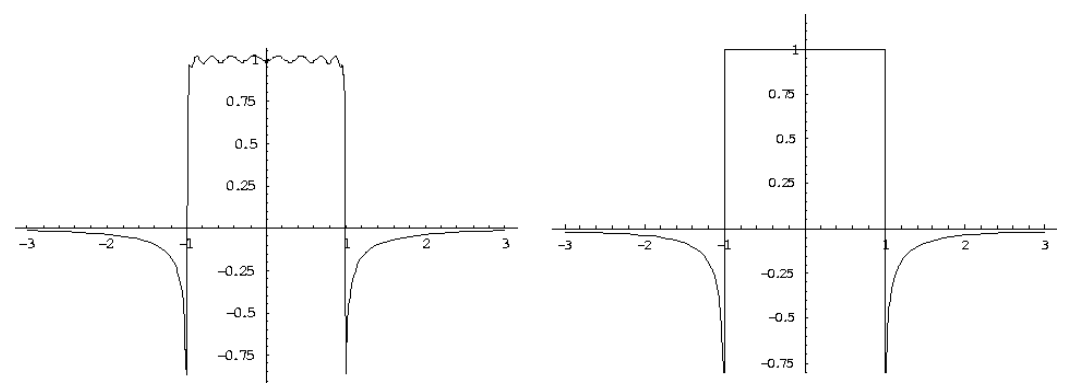

Fig.2. The left figure is the plot of a numerical solution for the leading density $\pi \sigma^{\ell}(u)$. The right figure represents an exact analytic solution for the same quantity.

We note that the functions $f_{k}(\theta)$ provide a complete set of functions on the interval $[0,2 \pi]$, while on the interval $[0, \pi]$ they are overcomplete. This fact is consistent with the ambiguity for the coefficients $s_{k}$ we observed in the previous section. Moreover, 
it is not hard to convince ourselves that the proposal for $s_{k}^{\ell}$ discussed in the previous section is the only possible solution such that $s_{2 k-1}^{\ell}=s_{2 k}^{\ell}$ and for which the density $\sigma^{\ell}(u)$ is constant for $|u| \leq 1$.

Further, it is easy to sum up the series defining the leading density for $|u|>1$. The result is

$$
\sigma^{\ell}(u)=\frac{1}{2 \pi}\left(2-\frac{\sqrt{2}}{\sqrt{1-u\left(u \mp \sqrt{u^{2}-1}\right)}}\right) .
$$

Here the minus and plus signs in the denominator corresponds to the regions $u>1$ and $u<-1$ respectively. The plot of the complete analytic solution for $\sigma^{\ell}(u)$ is presented in Fig.2. It should be compared to the plot of the numerical solution obtained by using in the series representation for the density the coefficients $s_{k}^{\ell}$ obtained from our numerical analysis. The numerical plot corresponds to taking $g=40$ and truncating the series at $k=20$.

Finally, we mention the expression for the scaling function $f(g)$ in terms of the density $\sigma(u)$ :

$$
f(g)=32 g^{3} \int_{-\infty}^{\infty} \mathrm{d} u \sigma(u)=8 g \int_{-\infty}^{\infty} \mathrm{d} u \sigma^{\ell}(u)+\ldots=4 g+\ldots
$$

This completes our discussion of the leading order analytic solution of the BES equation in the strong coupling limit.

\subsection{Subleading corrections}

Here we will investigate the first subleading correction to the leading coefficients $s_{k}^{\ell}$. By expanding the BES equation we have already obtained equation (2.24) which expresses the subleading density $s_{n}^{s \ell}$ in terms of the leading one:

$$
Z_{2 m-1, r}^{\ell} s_{r}^{s \ell}=-Z_{2 m-1, r}^{s \ell} s_{r}^{\ell}-\frac{1}{2(2 m-1)} s_{2 m-1}^{\ell}
$$

Again this equation is degenerate and needs to be supplemented by a second one that appears at higher order in $1 / g$ in the BES equation. Substituting the explicit form of the coefficients $s_{n}^{\ell}$ obtained above, the right hand side of eq. (2.36) is seen to vanish (i.e. $K^{(c) \ell} s^{s \ell}=0$ ), which already implies tight restrictions on the subleading corrections $s^{s \ell}$. As discussed above, this equation allows for solving for half of the components. For instance, using eqs.(2.14) and (2.17) we can solve for the even components in terms of the odd ones

$$
s_{2 m}^{s \ell}=-\sum_{n=1}^{\infty}(-1)^{m-n} \frac{32}{\pi} \frac{m^{3}}{\left(4 m^{2}-(1-2 n)^{2}\right)(1-2 n)^{2}} s_{2 n-1}^{s \ell} .
$$


To obtain another equation constraining the subleading solution, we examine corrections to the BES equation as follows. From the $\mathcal{O}(1 / g)$ contribution to the even rows of the BES equation $(\overline{2.20})$ we find

$$
\begin{aligned}
& s_{2 n}^{\ell}=8 n Z_{2 n, 1}^{s s \ell}-4 n Z_{2 n, m}^{\ell} s_{m}^{s \ell}-4 n Z_{2 n, m}^{s \ell} s_{m}^{\ell} \\
& -16 n(2 m-1)\left[Z_{2 n, 2 m-1}^{\ell} Z_{2 m-1, r}^{\ell} s_{r}^{s s \ell}+Z_{2 n, 2 m-1}^{\ell} Z_{2 m-1, r}^{s \ell} s_{r}^{s \ell}+Z_{2 n, 2 m-1}^{\ell} Z_{2 m-1, r}^{s s \ell} s_{r}^{\ell}\right. \\
& \left.\quad+Z_{2 n, 2 m-1}^{s \ell} Z_{2 m-1, r}^{\ell} s_{r}^{s \ell}+Z_{2 n, 2 m-1}^{s \ell} Z_{2 m-1, r}^{s \ell} s_{r}^{\ell}+Z_{2 n, 2 m-1}^{s s \ell} Z_{2 m-1, r}^{\ell} s_{r}^{\ell}\right] .
\end{aligned}
$$

To eliminate the term in $s^{s s \ell}$ we turn to the odd rows of the $\mathcal{O}\left(1 / g^{2}\right)$ equation

$$
-2(2 m-1)\left[Z_{2 m-1, r}^{\ell} s_{r}^{s s \ell}+Z_{2 m-1, r}^{s \ell} s_{r}^{s \ell}+Z_{2 m-1, r}^{s s \ell} s_{r}^{\ell}\right]=s_{2 m-1}^{s \ell} .
$$

Using this together with (2.22) and (2.24) to simplify (2.38) we obtain

$$
Z_{2 n, m}^{\ell}(-1)^{m} s_{m}^{s \ell}=-Z_{2 n, m}^{s \ell}(-1)^{m} s_{m}^{\ell}-\frac{1}{4 n} s_{2 n}^{\ell} .
$$

This can be combined with (2.36) into the full rank equation

$$
\tilde{Z}_{n m}^{\ell} s_{m}^{s \ell}=-\tilde{Z}_{n m}^{s \ell} s_{m}^{\ell}-\frac{1}{2 n} s_{n}^{\ell}
$$

where again the right hand side vanishes when evaluated on the leading solution $s^{\ell}$ found above.

In view of the divergence of the $\mathcal{O}(1 / g)$ correction to $Z_{n m}$, which could indicate the presence in it of terms e.g. $\mathcal{O}(\log (g) / g)$, the strong coupling expansion of $s_{n}(g)$ may contain more than just pure powers of $1 / g$. This complication begins at $\mathcal{O}\left(1 / g^{3}\right)$ in $s_{n}$ and suggests that the equations for the subleading terms have to be supplemented by additional constraints. Luckily, the equations for the leading term in section 2.3 are not affected by this complication. We hope to analyze the structure of subleading terms more fully in future work.

\section{Leading density from the integral equation}

In the appendix we compute the inverse Fourier transform of the BES equation (rather than that of its leading solution) to the rapidity variable $u .^{3}$ In this section we will expand the BES integral equation in the small parameter $\epsilon=1 /(2 g)$ assuming that the density expands as in eq.(2.31) and rederive the leading order solution $\sigma^{\ell}(u)$ by different means.

It turns out that the branch cut of the square root functions defining the allloops Bethe-ansatz [9] forces us to distinguish the regimes $|u| \gtrless 1$. The leading order

\footnotetext{
${ }^{3}$ This approach to the BES equation was also pursued by A. Belitsky (seminar at Princeton University, December 2006).
} 
BES equation constrains the leading density $\sigma^{\ell}(u)$ only within the unit interval; no condition is obtained for $|u|>1$. We will show that this equation has a unique solution, namely the constant function $\sigma^{\ell}(u)=1 / \pi,|u|<1$.

In the discussion of the last section, the even and the odd functions, $f_{2 k}$ and $f_{2 k-1}$, constitute equivalent complete sets of functions on the interval $|u| \leq 1$. The constraint that the density be constant in this region allows one to eliminate, e.g., the coefficients $s_{2 k-1}^{\ell}$ in favour of $s_{2 k}^{\ell}$ (or vice versa). Now, to leading order only the even functions $f_{2 k}$ have support outside the interval $|u|<1$. The knowledge of the density outside this interval would then fix the remaining coefficients $s_{2 k}^{\ell}$.

Above, we have rather taken the opposite approach: pairing the numerical observation that $s_{2 k-1}^{\ell}=s_{2 k}^{\ell}$ with the requirement that the density be constant within the unit interval we could equivalently fix the density $\sigma^{\ell}(u)$ on the whole real axis, c.f. eq.(2.35).

The leading terms in the strong coupling expansion of the BES equation are obtained in appendix 5.2. We find

$$
\int_{-1}^{1} \mathrm{~d} u^{\prime} \sigma^{\ell}\left(u^{\prime}\right) \hat{K}^{(c) \ell}\left(u, u^{\prime}\right)=2 \sqrt{1-u^{2}}-\frac{1}{\sqrt{1-u^{2}}},
$$

where

$$
\hat{K}^{(c) \ell}\left(u, u^{\prime}\right)=-2\left(\frac{\sqrt{1-\left(u^{\prime}\right)^{2}}}{\sqrt{1-u^{2}}}+\frac{1}{4} \log \left(\frac{\left(\sqrt{1-u^{2}}-\sqrt{1-\left(u^{\prime}\right)^{2}}\right)^{2}}{\left(\sqrt{1-u^{2}}+\sqrt{1-\left(u^{\prime}\right)^{2}}\right)^{2}}\right)\right) .
$$

In Fourier space, the potential for the BES equation is actually the kernel with the second argument put to zero; or to put it differently, the kernel integrated on a delta function. In the rapidity plane this means that the potential is the integral of the kernel on a constant function. By construction, our leading equation is solved by

$$
\sigma^{\ell}(u)=\frac{1}{\pi}, \quad|u|<1 .
$$

Let us check whether there is a second solution inside the interval $|u|<1$. To this end, we put

$$
U=\sqrt{1-u^{2}}, \quad V=\sqrt{1-\left(u^{\prime}\right)^{2}}
$$

and differentiate the whole equation in $U$. Next, we substitute $U^{2} \rightarrow U$ and $V^{2} \rightarrow V$ to obtain

$$
1+\frac{1}{2 U}=\int_{0}^{1} \mathrm{~d} V \tilde{\sigma}(V)\left(\frac{1}{U}-\frac{1}{U-V}\right),
$$

where $\tilde{\sigma}$ is the density written in the new variables but including the transformation of the integration measure:

$$
\tilde{\sigma}=\sigma \frac{\sqrt{V}}{\sqrt{1-V}}
$$


There is no regular density that could yield $1 / U$ from the Hilbert transform (i.e. the second term on the r.h.s.). We will therefore look for a density that produces the constant term on the l.h.s. from the Hilbert transform, and whose norm is defined by the $1 / U$ terms. Now,

$$
\frac{1}{\pi} \int_{0}^{1} \mathrm{~d} V \frac{\sqrt{V}}{\sqrt{1-V}} \frac{1}{U-V}=-1, \quad-\frac{1}{\pi} \int_{0}^{1} \mathrm{~d} V \frac{\sqrt{1-V}}{\sqrt{V}} \frac{1}{U-V}=-1
$$

and in the first case the normalization is $1 / 2$, while in the second it is $-1 / 2$. Note that the symmetric combination of the two trial densities

$$
\frac{\sqrt{V}}{\sqrt{1-V}}+\frac{\sqrt{1-V}}{\sqrt{V}}=\frac{1}{\sqrt{V} \sqrt{1-V}}
$$

goes to zero under the Hilbert transform whereas it does affect the norm. Thus, a solution exists and it is unique. We have to choose

$$
\tilde{\sigma}(V)=\frac{1}{\pi} \frac{\sqrt{V}}{\sqrt{1-V}}
$$

which corresponds to $\sigma\left(u^{\prime}\right)=1 / \pi$.

In appendix 5.2. we further derive the next-to-leading order of the BES equation. We find

$$
\int_{-1}^{1} \mathrm{~d} u^{\prime} \sigma^{s \ell}\left(u^{\prime}\right) \hat{K}^{(c) \ell}\left(u, u^{\prime}\right)=0, \quad:|u|<1 .
$$

By what was said above the leading dressing kernel is invertible on $[-1,1]$. We therefore conclude

$$
\sigma^{s \ell}(u)=0, \quad|u|<1 .
$$

No constraint is found on the subleading density outside the unit interval.

We refrain from pushing the analysis any further for the following reasons: First, at the next order we would perhaps encounter the same degeneracy while we lack an independent condition like $s_{2 k}^{\ell}=s_{2 k-1}^{\ell}$ that would allow us to make progress. Second, the naive expansion in $\epsilon$ quickly leads to expressions with rather hard singularities in $u, u^{\prime}$ which are difficult to handle consistently.

The original BES equation has the property that the energy is given by the value of the density at zero. In the rapidity variables this means that we can recover the leading contribution to the energy from the normalization of $\sigma^{\ell}$. Alternatively, we 
may use the formula

$$
\begin{aligned}
& E(g)=\log (S) 8 g^{2} {\left[1-2 g^{2} \int_{-\infty}^{\infty} \mathrm{d} u \sigma(u)\left(\frac{i}{x^{+}(u)}-\frac{i}{x^{-}(u)}\right)\right] } \\
&=\frac{2 \log (S)}{\epsilon^{2}}\left[1-\frac{2}{\epsilon^{2}} \int_{0}^{1} \mathrm{~d} u \sigma(u)\left(2 \sqrt{1-u^{2}}-\epsilon+\ldots\right)-\right. \\
&\left.-\frac{2}{\epsilon^{2}} \int_{1}^{\infty} \mathrm{d} u \sigma(u)\left(\frac{\epsilon}{u^{2}\left(1+\sqrt{1-\frac{1}{u^{2}}}\right)-1}+\ldots\right)\right] .
\end{aligned}
$$

It is instructive to see how the value of the energy predicted from string theory is reproduced: The contribution of the constant part of $\sigma^{\ell}$ within the unit interval cancels the leading 1 in the square bracket. On the other hand, the next-to-leading term would receive a contribution from an a priory non-vanishing subleading density inside the unit interval. We get

$$
\begin{aligned}
E(g)=\log (S) 8 g\left(\int_{0}^{1} \mathrm{~d} u\right. & \sigma^{\ell}(u)-\int_{1}^{\infty} \mathrm{d} u \frac{\sigma^{\ell}(u)}{u^{2}\left(1+\sqrt{1-\frac{1}{u^{2}}}\right)-1} \\
& \left.-2 \int_{0}^{1} \mathrm{~d} u \sigma^{s \ell}(u) \sqrt{1-u^{2}}\right)+\ldots=\log (S) 4 g+\ldots
\end{aligned}
$$

Here we used an identity

$$
\int_{0}^{1} \mathrm{~d} u \sigma^{\ell}(u)-\int_{1}^{\infty} \mathrm{d} u \frac{\sigma^{\ell}(u)}{u^{2}\left(1+\sqrt{1-\frac{1}{u^{2}}}\right)-1}=\int_{-\infty}^{\infty} \mathrm{d} u \sigma^{\ell}(u) .
$$

satisfied by the leading density and also the fact that the subleading density vanishes inside the unit interval. In fact, the absence of the subleading density can be considered as the consistency test on our equations.

\section{Conclusions}

In this paper we analyze the strong coupling limit of the BES equation which describes the universal scaling function of high spin operators in $\mathcal{N}=4$ gauge theory. We have shown that expanding the BES equation in inverse powers of the coupling constant leads to two equations for the large $g$ solution, one appearing at leading the other at subleading order in $1 / \mathrm{g}$. Together they determine a unique solution in the strong coupling limit whose exact analytic form we present.

Obviously, the next step would be to understand the structure of the higher order perturbation theory around the strong coupling point and, in particular, to derive

the next-to-leading corrections to the universal scaling function $f(g)$. We make some 
progress in this direction by deriving a pair of equations for the subleading solution, but also point out some subtleties that arise in developing the expansion around the leading order solution.

As was shown in [10] (see also [24]), the coefficients of the perturbative series describing the solution of the BES equation at weak coupling admit an analytic continuation to strong coupling, where they coincide with those predicted by string theory. The approach we adopt here can be considered as another, complementary way to analytically continue from weak to strong coupling.

On the rapidity $u$-plane the leading fluctuation density $\sigma^{\ell}(u)$ appears to be constant inside the unit interval $|u|<1$. We could argue that this constant part of $\sigma^{\ell}(u)$ is an artifact of the way the BES equation was derived: The non-vanishing constant part of the leading density offsets the splitting of the weak-coupling density into a $\log$ divergent one-loop part and a regular higher loop piece carrying $\log S$ as a coefficient. Further, as we have shown, the subleading correction inside the unit interval is absent; in a manner of speaking a gap opens between $[-\infty,-1]$ and $[1, \infty]$. This could be qualitatively compared to the results obtained from string theory. Indeed, the solution of the integral equation describing the classical spinning strings in $\mathrm{AdS}_{3} \times \mathrm{S}^{1}$ [25] in the limit $S \rightarrow \infty$ with spin $J$ along $\mathrm{S}^{1}$ fixed has support only outside the interval $|u|<1$. The same behavior is expected ${ }^{4}$ for the GKP solution which is obtained in the limit $J \rightarrow 0$. For finite $S$ the solution is elliptic and it exhibits logarithmic singularities in the limit $S \rightarrow \infty$. On the other hand, our strong coupling density (2.35) is an algebraic function which carries $\log S$ as a normalization. Of course, this density leads to the same energy as for the GKP string. Thus, it is desirable to understand the detailed matching between the string density (higher conserved charges) and the density we found from the strong coupling limit of the BES equation. We plan to return to this interesting question elsewhere.

\section{Acknowledgements}

We are grateful to Andrei Belitsky, Sergey Frolov and Matthias Staudacher for valuable discussions. I. R. K. and M. K. B. would like to thank Sergio Benvenuti and Antonello Scardicchio for collaboration on the early stages of this project. This work was supported in part by the EU-RTN network Constituents, Fundamental Forces and Symmetries of the Universe (MRTN-CT-2004-005104), by the INTAS contract 03-51-6346, by the NWO grant 047017015, and by the National Science Foundation under grant No. PHY-0243680. The work of L. F. A. was supported by the VENI grant 680-47-113. The work of G. A. was supported in part by the RFBI grant N05-01-00758 and by the grant NSh-672.2006.1.

\footnotetext{
${ }^{4}$ We would like to thank Sergey Frolov for the discussion of this point.
} 


\section{Appendix}

\subsection{The inverse Fourier transform of the BES equation}

Let us define the kernels

$$
\begin{aligned}
& K_{+}\left(u, u^{\prime}\right)=\frac{\left(1-\frac{g^{2}}{x^{+}(u) x^{-}\left(u^{\prime}\right)}\right)\left(1+\frac{g^{2}}{x^{+}(u) x^{-}\left(u^{\prime}\right)}\right)}{\left(1-\frac{g^{2}}{x^{-}(u) x^{+}\left(u^{\prime}\right)}\right)\left(1+\frac{g^{2}}{x^{-}(u) x^{-}\left(u^{\prime}\right)}\right)}, \\
& K_{-}\left(u, u^{\prime}\right)=\frac{\left(1+\frac{g^{2}}{x^{-}(u) x^{+}\left(u^{\prime}\right)}\right)\left(1-\frac{g^{2}}{x^{-}(u) x^{-}\left(u^{\prime}\right)}\right)}{\left(1+\frac{g^{2}}{x^{+}(u) x^{-}\left(u^{\prime}\right)}\right)\left(1-\frac{g^{2}}{x^{+}(u) x^{+}\left(u^{\prime}\right)}\right)},
\end{aligned}
$$

with

$$
x^{ \pm}(u)=\frac{1}{2}\left(u \pm \frac{i}{2}\right)\left(1+\sqrt{1-\frac{4 g^{2}}{\left(u \pm \frac{i}{2}\right)^{2}}}\right) .
$$

We further define

$$
\begin{aligned}
\hat{K}_{0}\left(u, u^{\prime}\right) & =\frac{i}{2} \partial_{u} \log \left(K_{+}\left(u, u^{\prime}\right) K_{-}\left(u, u^{\prime}\right)\right), \\
\hat{K}_{1}\left(u, u^{\prime}\right) & =\frac{i}{2} \partial_{u} \log \left(K_{+}\left(u, u^{\prime}\right) / K_{-}\left(u, u^{\prime}\right)\right) .
\end{aligned}
$$

In the same way as in appendix D of [5] we may show that

$$
\hat{K}_{0,1}\left(u, u^{\prime}\right)=2 g^{2} \iint_{-\infty}^{\infty} \mathrm{d} t \mathrm{~d} t^{\prime} e^{i u t+i u^{\prime} t^{\prime}}|t| e^{-\left(|t|+\left|t^{\prime}\right|\right) / 2} K_{0,1}\left(2 g|t|, 2 g\left|t^{\prime}\right|\right)
$$

where $K_{0,1}$ are defined in (2.4) in the main text. The dressing kernel is 10

$$
K^{(c)}\left(2 g|t|, 2 g\left|t^{\prime}\right|\right)=2 g^{2} \int_{-\infty}^{\infty} \mathrm{d} t^{\prime \prime} K_{1}\left(2 g|t|, 2 g\left|t^{\prime \prime}\right|\right) \frac{\left|t^{\prime \prime}\right|}{e^{\left|t^{\prime \prime}\right|}-1} K_{0}\left(2 g\left|t^{\prime \prime}\right|, 2 g\left|t^{\prime}\right|\right)
$$

and its Fourier back-transform yields

$$
\begin{aligned}
\hat{K}^{(c)}\left(u, u^{\prime}\right) & =2 g^{2} \iint_{-\infty}^{\infty} \mathrm{d} t \mathrm{~d} t^{\prime} e^{i u t+i u^{\prime} t^{\prime}}|t| e^{-\left(|t|+\left|t^{\prime}\right|\right) / 2} K^{(c)}\left(2 g|t|, 2 g\left|t^{\prime}\right|\right) \\
& =\frac{1}{4 \pi^{2}} \iiint_{-\infty}^{\infty} \mathrm{d} v \mathrm{~d} v^{\prime} \mathrm{d} t^{\prime \prime} \hat{K}_{0}(u, v) \frac{e^{-i\left(v+v^{\prime}\right) t^{\prime \prime}}}{1-e^{-\left|t^{\prime \prime}\right|}} \hat{K}_{1}\left(v^{\prime}, u^{\prime}\right) .
\end{aligned}
$$

In the $u, u^{\prime}$ variables the BES equation becomes:

$$
\begin{aligned}
0 & =2 \pi \sigma(u)-2 \int_{-\infty}^{\infty} \mathrm{d} u^{\prime} \sigma\left(u^{\prime}\right) \frac{1}{\left(u-u^{\prime}\right)^{2}+1} \\
& +\int_{-\infty}^{\infty} \mathrm{d} u^{\prime}\left(\sigma\left(u^{\prime}\right)-\frac{1}{4 \pi g^{2}}\right)\left(\hat{K}_{0}\left(u, u^{\prime}\right)+\hat{K}_{1}\left(u, u^{\prime}\right)+2 \hat{K}^{(c)}\left(u, u^{\prime}\right)\right) .
\end{aligned}
$$

We have written the potential as the integral of the kernels on the constant function $-1 /\left(4 \pi g^{2}\right)$, i.e. the Fourier back-transform of $-1 /\left(2 g^{2}\right) \delta\left(t^{\prime}\right)$. 


\subsection{The strong coupling limit}

The integration over $t^{\prime \prime}$ in eq.(5.8) is not well defined. To make sense of it we borrow the derivative from $\hat{K}_{0}$ (c.f. (5.4)) on the right:

$$
\begin{aligned}
\partial_{v^{\prime}} \int_{-\infty}^{\infty} \mathrm{d} t^{\prime \prime} \frac{e^{-i\left(v+v^{\prime}\right) t^{\prime \prime}}}{1-e^{-\left|t^{\prime \prime}\right|}} & =-i \int_{-\infty}^{\infty} \mathrm{d} t^{\prime \prime} e^{-i\left(v+v^{\prime}\right) t^{\prime \prime}}\left(\frac{t^{\prime \prime}}{e^{\left|t^{\prime \prime}\right|}-1}+t^{\prime \prime}\right) \\
& =\partial_{v^{\prime}}\left(-\Psi\left(i\left(v+v^{\prime}\right)\right)-\Psi\left(-i\left(v+v^{\prime}\right)\right)+2 \pi \delta\left(v+v^{\prime}\right)\right),
\end{aligned}
$$

where $\Psi(v)$ is the digamma function. We may then partially integrate the outer derivative back to act on $\hat{K}_{0}$.

The physical situation we consider is in a kinematical regime in which $u$ scales with $2 g$ at strong coupling, as can be seen for instance from the numerical studies of the integral equation for density of fluctuations in [5]. In the following we will rescale $u \rightarrow 2 g u$ and consider a perturbation series in $\epsilon=1 /(2 g)$.

In the middle integral in eq.(5.8) we rescale and employ the asymptotic expansion of the digamma function:

$$
\begin{array}{r}
-\Psi\left(i\left(v+v^{\prime}\right)\right)-\Psi\left(-i\left(v+v^{\prime}\right)\right)+2 \pi \delta\left(v+v^{\prime}\right) \\
\rightarrow-\log \left(\left(v+v^{\prime}\right)^{2}\right)+\frac{\pi}{g} \delta\left(v+v^{\prime}\right)+\mathcal{O}\left(\epsilon^{2}\right)
\end{array}
$$

Note that we have discarded a term $\log (2 g)+c_{0}$, where $c_{0}$ is an integration constant from the differentiation trick. A constant integrated onto the leading order of $\hat{K}_{1}$ in the second argument (or $\hat{K}_{0}$ in the first) actually yields zero. Further, the derivative of the exact expression in terms of the digamma function is a principal value for $1 /\left(v+v^{\prime}\right)$ (i.e. a cut-off at zero). We usually evaluate $\log \left(\left(v+v^{\prime}\right)^{2}\right)$ under the subsequent $v, v^{\prime}$ integrations by partial integration, albeit using a Cauchy principal value. It ought not matter which definition is used for the principal value.

After rescaling we find the following leading order dressing kernel in configuration space:

$$
\hat{K}^{(c) \ell}\left(u, u^{\prime}\right)=-\frac{1}{2 \pi^{2}} \iint_{-\infty}^{\infty} \mathrm{d} v \mathrm{~d} v^{\prime} \tilde{K}_{1}^{\ell}(u, v)\left(\frac{1}{2} \log \left(\left(v+v^{\prime}\right)^{2}\right)\right) \tilde{K}_{0}^{\ell}\left(v^{\prime}, u^{\prime}\right) .
$$

Here $\hat{K}_{0,1}=\epsilon \tilde{K}_{0,1}$. To leading order

$$
\begin{aligned}
|u|>1: x^{+}(u) & =x^{-}(u)=\frac{1}{2 \epsilon} u\left(1+\sqrt{1-\frac{1}{u^{2}}}\right)+\ldots \\
|u|<1: x^{+}(u) & =\frac{1}{2 \epsilon}\left(u+i \sqrt{1-u^{2}}\right)+\ldots \\
x^{-}(u) & =\frac{1}{2 \epsilon}\left(u-i \sqrt{1-u^{2}}\right)+\ldots
\end{aligned}
$$


from which it is easy to obtain

$$
\tilde{K}_{0}^{\ell}(u, v)=0 \quad: \quad|v|>1, \quad \tilde{K}_{1}^{\ell}(u, v)=0 \quad: \quad|u|>1
$$

and the non-vanishing sectors

$$
\begin{aligned}
& |u|<1: \tilde{K}_{0}^{\ell}(u, v)=\pi(\delta(u-v)+\delta(u+v)), \\
& |u|>1: \tilde{K}_{0}^{\ell}(u, v)=-2 \frac{\sqrt{1-v^{2}}}{\sqrt{1-\frac{1}{u^{2}}}} \frac{1}{u^{2}-v^{2}}
\end{aligned}
$$

and

$$
\begin{aligned}
& |v|<1: \tilde{K}_{1}^{\ell}(u, v)=\pi(\delta(u-v)+\delta(u+v))-\frac{2}{\sqrt{1-u^{2}}}, \\
& |v|>1: \tilde{K}_{1}^{\ell}(u, v)=-\frac{2}{\sqrt{1-u^{2}}}\left(1+\sqrt{1-\frac{1}{v^{2}}} \frac{v^{2}}{u^{2}-v^{2}}\right) .
\end{aligned}
$$

In particular, the leading contribution (5.12) to $\hat{K}^{(c)}\left(u, u^{\prime}\right)$ is only non-vanishing when both $u, u^{\prime}$ lie in the unit interval. Let us evaluate eq.(5.12) explicitly, plugging in the formulae for $\tilde{K}_{0,1}^{\ell}$ and executing the two integrations in turn. First,

$$
\begin{aligned}
& \left(\int_{-\infty}^{-1}+\int_{1}^{\infty}\right) \mathrm{d} v^{\prime} \frac{1}{2} \log \left(\left(v+v^{\prime}\right)^{2}\right) \tilde{K}_{0}^{\ell}\left(v^{\prime}, u^{\prime}\right) \\
= & -2 \pi \log \left(\sqrt{1-\left(u^{\prime}\right)^{2}}+\sqrt{1-v^{2}}\right) \quad: \quad\left|u^{\prime}\right|,|v|<1 \\
= & -\pi \log \left(v^{2}-\left(u^{\prime}\right)^{2}\right) \quad: \quad\left|u^{\prime}\right|<1,|v|>1,
\end{aligned}
$$

while the $v^{\prime}$-integration over the unit interval contributes

$$
\int_{-1}^{1} \mathrm{~d} v^{\prime} \frac{1}{2} \log \left(\left(v+v^{\prime}\right)^{2}\right) \tilde{K}_{0}^{\ell}\left(v^{\prime}, u^{\prime}\right)=\frac{\pi}{2} \log \left(\left(v^{2}-\left(u^{\prime}\right)^{2}\right)^{2}\right) .
$$

Hence, if $\left|u^{\prime}\right|,|v|<1$

$$
\begin{aligned}
& \int_{-\infty}^{\infty} \mathrm{d} v^{\prime} \frac{1}{2} \log \left(\left(v+v^{\prime}\right)^{2}\right) \tilde{K}_{0}^{\ell}\left(v^{\prime}, u^{\prime}\right) \\
= & -2 \pi \log \left(\sqrt{1-\left(u^{\prime}\right)^{2}}+\sqrt{1-v^{2}}\right)+\frac{\pi}{2} \log \left(\left(v^{2}-\left(u^{\prime}\right)^{2}\right)^{2}\right) \\
= & \frac{\pi}{2} \log \left(\frac{\left(\sqrt{1-v^{2}}-\sqrt{1-\left(u^{\prime}\right)^{2}}\right)^{2}}{\left(\sqrt{1-v^{2}}+\sqrt{1-\left(u^{\prime}\right)^{2}}\right)^{2}}\right)
\end{aligned}
$$

and otherwise we find zero. It is then easy to see that

$$
\hat{K}^{(c) \ell}\left(u, u^{\prime}\right)=-2\left(\frac{\sqrt{1-\left(u^{\prime}\right)^{2}}}{\sqrt{1-u^{2}}}+\frac{1}{4} \log \left(\frac{\left(\sqrt{1-u^{2}}-\sqrt{1-\left(u^{\prime}\right)^{2}}\right)^{2}}{\left(\sqrt{1-u^{2}}+\sqrt{1-\left(u^{\prime}\right)^{2}}\right)^{2}}\right)\right)
$$


to leading order in the strong coupling expansion.

The rescaled BES equation is

$$
\begin{aligned}
0 & =2 \pi \sigma(u)-2 \int_{-\infty}^{\infty} \mathrm{d} u^{\prime} \sigma\left(u^{\prime}\right) \frac{\epsilon}{\left(u-u^{\prime}\right)^{2}+\epsilon^{2}} \\
& +\int_{-\infty}^{\infty} \mathrm{d} u^{\prime}\left(\sigma\left(u^{\prime}\right)-\frac{\epsilon^{2}}{\pi}\right)\left(\tilde{K}_{0}\left(u, u^{\prime}\right)+\tilde{K}_{1}\left(u, u^{\prime}\right)+\frac{2}{\epsilon} \hat{K}^{(c)}\left(u, u^{\prime}\right)\right) .
\end{aligned}
$$

Note that the kernel in the second term on the r.h.s. is a representation of $\pi \delta\left(u-u^{\prime}\right)$ so that the first two terms cancel for small $\epsilon$. The numerical analysis displayed in the paper suggests to look for a solution

$$
\sigma\left(u^{\prime}\right)=\epsilon^{2} \sigma^{\ell}\left(u^{\prime}\right)+\epsilon^{3} \sigma^{s \ell}\left(u^{\prime}\right)+\mathcal{O}\left(\epsilon^{4}\right)
$$

The leading equation is

$$
\int_{-1}^{1} \mathrm{~d} u^{\prime}\left(\sigma^{\ell}\left(u^{\prime}\right)-\frac{1}{\pi}\right) \hat{K}^{(c) \ell}\left(u, u^{\prime}\right)=0 .
$$

It constrains the density $\sigma^{\ell}$ only inside the unit interval. We prove in the main text that the obvious solution

$$
\sigma^{\ell}\left(u^{\prime}\right)=\frac{1}{\pi} \quad: \quad\left|u^{\prime}\right|<1
$$

is also the only one. At the next order we find the equation

$$
\begin{aligned}
0 & =\left(\int_{-\infty}^{-1}+\int_{1}^{\infty}\right) \mathrm{d} u^{\prime}\left(\sigma^{\ell}\left(u^{\prime}\right)-\frac{1}{\pi}\right)\left(\tilde{K}_{0}^{\ell}\left(u, u^{\prime}\right)+\tilde{K}_{1}^{\ell}\left(u, u^{\prime}\right)+2 \hat{K}^{(c) s \ell}\left(u, u^{\prime}\right)\right) \\
& +\int_{-1}^{1} \mathrm{~d} u^{\prime} \sigma^{s \ell}\left(u^{\prime}\right) \hat{K}^{(c) \ell}\left(u, u^{\prime}\right) .
\end{aligned}
$$

Now, to leading order $\tilde{K}_{0}\left(u, u^{\prime}\right)$ vanishes when $\left|u^{\prime}\right|>1$ so that the $\tilde{K}_{0}^{\ell}$ term can be dropped. We show below that the other two kernels in the first line cancel. Hence for any $\sigma^{\ell}$ the last equation reduces to

$$
\int_{-1}^{1} \mathrm{~d} u^{\prime} \sigma^{s \ell}\left(u^{\prime}\right) \hat{K}^{(c) \ell}\left(u, u^{\prime}\right)=0 .
$$

It follows that

$$
\sigma^{s \ell}\left(u^{\prime}\right)=0 \quad: \quad\left|u^{\prime}\right|<1
$$

because $\hat{K}^{(c) s \ell}$ is invertible on the unit interval (see Section 3). At this stage we cannot make a statement about $\sigma^{s \ell}$ outside the unit interval.

To show the aforementioned cancellation of the two kernels, let us work out $\hat{K}^{(c) s \ell}\left(u, u^{\prime}\right)$ for $\left|u^{\prime}\right|>1$. In this case, $\tilde{K}_{0}^{\ell}\left(v^{\prime}, u^{\prime}\right)$ in (5.12) vanishes, so that we pick 
up the subleading correction in the right factor while the other two terms may still be taken at leading order. From eq. (5.4) we can work out

$$
\begin{aligned}
& |u|<1,\left|u^{\prime}\right|>1: \tilde{K}_{0}^{s \ell}\left(u, u^{\prime}\right)=\frac{u^{2}+\left(u^{\prime}\right)^{2}}{\left(u^{2}-\left(u^{\prime}\right)^{2}\right)^{2}} \\
& |u|>1,\left|u^{\prime}\right|>1: \tilde{K}_{0}^{s \ell}\left(u, u^{\prime}\right)=\frac{1-u^{2}-\left(u^{\prime}\right)^{2}}{\Delta},
\end{aligned}
$$

where we have introduced the notation

$$
\begin{aligned}
\Delta= & \left(u^{2}\left(u^{\prime}\right)^{2}+\left|u u^{\prime}\right| \sqrt{u^{2}-1} \sqrt{\left(u^{\prime}\right)^{2}-1}\right)\left(-2+u^{2}+\left(u^{\prime}\right)^{2}\right) \\
& -u^{2}\left(u^{2}-1\right)-\left(u^{\prime}\right)^{2}\left(\left(u^{\prime}\right)^{2}-1\right) .
\end{aligned}
$$

It is not too hard to check that

$$
\begin{aligned}
& \int_{-\infty}^{\infty} \frac{1}{2} \log \left(\left(v+v^{\prime}\right)^{2}\right) \tilde{K}_{0}^{s \ell}\left(v^{\prime}, u^{\prime}\right) \\
= & -\frac{\pi}{\left(u^{\prime}\right)^{2}-v^{2}} \frac{\sqrt{1-v^{2}}}{\sqrt{1-\frac{1}{\left(u^{\prime}\right)^{2}}}} \quad: \quad|v|<1,\left|u^{\prime}\right|>1, \\
= & 0 \quad: \quad|v|,\left|u^{\prime}\right|>1
\end{aligned}
$$

from which we may easily deduce that

$$
\hat{K}^{(c) s \ell}\left(u, u^{\prime}\right)=-\frac{1}{2} \tilde{K}_{1}^{\ell}\left(u, u^{\prime}\right) \quad: \quad|u|<1,\left|u^{\prime}\right|>1 .
$$

\section{References}

[1] A. V. Kotikov and L. N. Lipatov, "DGLAP and BFKL evolution equations in the N = 4 supersymmetric gauge theory," hep-ph/0112346; A. V. Kotikov, L. N. Lipatov and V. N. Velizhanin, "Anomalous dimensions of Wilson operators in N = 4 SYM theory," Phys. Lett. B 557 (2003) 114, hep-ph/0301021.

[2] S. S. Gubser, I. R. Klebanov and A. M. Polyakov, "A semi-classical limit of the gauge/string correspondence," Nucl. Phys. B 636 (2002) 99, hep-th/0204051.

[3] S. Frolov and A. A. Tseytlin, "Semiclassical quantization of rotating superstring in $\mathrm{AdS}_{5} \times \mathrm{S}^{5}$, , JHEP 0206 (2002) 007, hep-th/0204226.

[4] A. V. Belitsky, A. S. Gorsky and G. P. Korchemsky, "Logarithmic scaling in gauge / string correspondence," Nucl. Phys. B 748 (2006) 24, hep-th/0601112.

[5] B. Eden and M. Staudacher, "Integrability and transcendentality," J. Stat. Mech. 0611 (2006) P014, hep-th/0603157.

[6] N. Beisert, V. Dippel and M. Staudacher, "A novel long range spin chain and planar $\mathcal{N}=4$ super Yang-Mills," JHEP 0407 (2004) 075, hep-th/0405001. 
[7] G. Arutyunov, S. Frolov and M. Staudacher, "Bethe ansatz for quantum strings," JHEP 0410 (2004) 016, hep-th/0406256.

[8] M. Staudacher, "The factorized S-matrix of CFT/AdS," JHEP 0505 (2005) 054, hep-th/0412188.

[9] N. Beisert and M. Staudacher, "Long-range PSU $(2,2 \mid 4)$ Bethe ansaetze for gauge theory and strings," Nucl. Phys. B 727 (2005) 1, hep-th/0504190.

[10] N. Beisert, B. Eden and M. Staudacher, "Transcendentality and crossing," J. Stat. Mech. 0701 (2007) P021, hep-th/0610251.

[11] Z. Bern, M. Czakon, L. J. Dixon, D. A. Kosower and V. A. Smirnov, "The four-loop planar amplitude and cusp anomalous dimension in maximally supersymmetric Yang-Mills theory," hep-th/0610248.

[12] F. Cachazo, M. Spradlin and A. Volovich, "Four-loop cusp anomalous dimension from obstructions," hep-th/0612309.

[13] N. Beisert, "The $\mathfrak{s u ( 2 | 2 ) ~ d y n a m i c ~ S - m a t r i x , " ~ h e p - t h / 0 5 1 1 0 8 2 . ~}$

[14] D. M. Hofman and J. M. Maldacena, "Giant magnons," J. Phys. A 39 (2006) 13095, hep-th/0604135; J. Maldacena and I. Swanson, "Connecting giant magnons to the pp-wave: An interpolating limit of $\mathrm{AdS}_{5} \times \mathrm{S}^{5}$," hep-th/0612079.

[15] T. Klose, T. McLoughlin, R. Roiban and K. Zarembo, "Worldsheet scattering in $\mathrm{AdS}_{5} \times \mathrm{S}^{5}, "$ hep-th/0611169.

[16] G. Arutyunov, S. Frolov and M. Zamaklar, "The Zamolodchikov-Faddeev algebra for $\mathrm{AdS}_{5} \times \mathrm{S}^{5}$ superstring," hep-th/0612229.

[17] R. A. Janik, "The $\mathrm{AdS}_{5} \times \mathrm{S}^{5}$ superstring worldsheet S-matrix and crossing symmetry," Phys. Rev. D 73 (2006) 086006, hep-th/0603038.

[18] G. Arutyunov and S. Frolov, "On $\mathrm{AdS}_{5} \times \mathrm{S}^{5}$ string S-matrix," Phys. Lett. B 639 (2006) 378, hep-th/0604043.

[19] R. Hernandez and E. Lopez, "Quantum corrections to the string Bethe ansatz," JHEP 0607 (2006) 004, hep-th/0603204.

[20] N. Beisert, R. Hernandez and E. Lopez, "A crossing-symmetric phase for $\mathrm{AdS}_{5} \times \mathrm{S}^{5}$ strings," JHEP 0611 (2006) 070, hep-th/0609044.

[21] J. M. Maldacena, "The large N limit of superconformal field theories and supergravity," Adv. Theor. Math. Phys. 2 (1998) 231 [Int. J. Theor. Phys. 38 (1999) 1113] hep-th/9711200.

[22] M. K. Benna, S. Benvenuti, I. R. Klebanov and A. Scardicchio, "A test of the AdS/CFT correspondence using high-spin operators," hep-th/0611135. 
[23] A. V. Kotikov and L. N. Lipatov, "On the highest transcendentality in $\mathrm{N}=4$ SUSY," hep-th/0611204.

[24] C. Gomez and R. Hernandez, "Integrability and non-perturbative effects in the AdS/CFT correspondence," Phys. Lett. B 644 (2007) 375, hep-th/0611014.

[25] V. A. Kazakov and K. Zarembo, "Classical / quantum integrability in non-compact sector of AdS/CFT," JHEP 0410 (2004) 060, hep-th/0410105. 